Check for updates

Cite this: RSC Adv., 2019, 9, 6871

Received 21st January 2019

Accepted 19th February 2019

DOI: $10.1039 / c 9 r a 00535 h$

rsc.li/rsc-advances

\section{Characterization and molecular docking of new $\Delta 17$ fatty acid desaturase genes from Rhizophagus irregularis and Octopus bimaculoides $\dagger$}

\author{
Chunchi Rong, ${ }^{\text {ab }}$ Haiqin Chen, (D) *ab Xin Tang, ${ }^{\text {ab }}$ Zhennan Gu, (D) ab Jianxin Zhao, ${ }^{\text {ab }}$ \\ Hao Zhang, ${ }^{\text {ab }}$ Wei Chen ${ }^{\text {abcd }}$ and Yong Q. Chen ${ }^{\text {abe }}$
}

Fatty acid desaturases are key enzymes in the biosynthesis of $n-3$ polyunsaturated fatty acids (PUFAs) via conversion of $n-6$ polyunsaturates to their $n-3$ counterparts. In this study, we reported the characterization and molecular docking of $\Delta 17$ desaturases from Rhizophagus irregularis and Octopus bimaculoides. These two new desaturase genes were screened using the known $\Delta 17$ desaturase gene (OPaFADS17) from Pythium aphanidermatum as a template. Analysis of their genes revealed that the sequences of oRiFADS17 and OObFADS17 contained the typical His-rich motifs (one HXXXH and two $\mathrm{HXXHH).} \mathrm{They} \mathrm{were} \mathrm{then} \mathrm{expressed} \mathrm{in} \mathrm{Saccharomyces} \mathrm{cerevisiae} \mathrm{INVSC1} \mathrm{to} \mathrm{examine} \mathrm{their} \mathrm{activities} \mathrm{and}$ substrate preferences. Our results show that the two candidate $n-3$ desaturases possess a strong $\Delta 17$ desaturase activity, exhibiting remarkable increase in desaturation activity on C20 fatty acids compared to $\mathrm{C} 18$ fatty acids. To the best of our knowledge, oRiFADS17 desaturase has greater (3-4 fold) catalytic activity for C18 substrates than other reported $\Delta 17$ desaturases and oObFADS17 is the first reported $\Delta 17$ desaturase in sea mollusks. Characterization of these two new desaturases will be of greater value for genetic engineering in industrial production of eicosapentaenoic acid (EPA, C20:5n-3) and docosahexaenoic acid (DHA, C22:6n-3). Due to lack of crystal structure information about $n-3$ desaturases, for the first time, the view of their predicted structures, binding pockets and substrate tunnels was clearly observed based on molecular docking. This will contribute to strengthening our understanding of the structure-function relationships of $n-3$ fatty acid desaturases.

\section{Introduction}

n-3 Polyunsaturated fatty acids (PUFAs) such as eicosapentaenoic acid (EPA, C20:5n-3) and docosahexaenoic acid (DHA, C22:6n-3) are important for brain development, tissue formation and repair in mammals., ${ }^{\mathbf{1} 2}$ They also have significant effects in the prevention of asthma, cancer, depression, obesity and immune cardiovascular disorder. ${ }^{3-5}$ However, the limited natural sources of $n$-3 PUFAs come mainly from microalgae oil and fish oil. ${ }^{6}$ As an alternative source, microorganisms have been used to produce EPA and DHA. ${ }^{7}$

${ }^{a}$ State Key Laboratory of Food Science and Technology, Jiangnan University, Wuxi, Jiangsu, P. R. China. E-mail: haiqinchen@jiangnan.edu.cn; Tel: +8651085197239 ${ }^{b}$ School of Food Science and Technology, Jiangnan University, Wuxi, Jiangsu, P. R. China

${ }^{c}$ National Engineering Research Center for Functional Food, Jiangnan University, Wuxi, Jiangsu, P. R. China

${ }^{d}$ Beijing Innovation Centre of Food Nutrition and Human Health, Beijing Technology and Business University (BTBU), Beijing, P. R. China

${ }^{e}$ Department of Cancer Biology, Wake Forest School of Medicine, Winston-Salem, NC, USA

$\dagger$ Electronic supplementary information (ESI) available. See DOI: 10.1039/c9ra00535h
The $n-6 / n-3$ fatty acid metabolic flux in microbial cells is tightly regulated by its intrinsic synthesis pathways $(\Delta 6 \mathrm{Des} /$ $\Delta 6$ Elo/n3Des or $\Delta 9$ Elo/ $\Delta 8$ Des/n3Des), and the key regulator n3Des is located at the branch point of metabolic flux. ${ }^{\mathbf{8} 9}$ It can convert $n$-6 PUFAs linoleic acid (LA, C18:2n-6), $\gamma$-linoleic acid (GLA, C18:3n-6), dihomo- $\gamma$-linoleic acid (DGLA, C20:3n-6) and arachidonic acid (AA, C20:4n-6) into $n$-3 PUFAs $\alpha$-linoleic acid (ALA, C18:3n-3), stearidonic acid (STA, C18:4n-3), eicosatetraenoic acid (ETA, C20:4n-3) and eicosapentaenoic acid (EPA, C20:4n-3), respectively, and is crucial for the synthesis of $n-3$ long-chain PUFAs (LC-PUFAs). ${ }^{\mathbf{1 0 , 1 1}}$

Some reports have involved studies of various types of n3Des from a wide range of species including microalgae, cyanobacteria, yeast, fungi and plants. ${ }^{6,7,12}$ n3Des from various species were shown to exhibit a broad $n-6$ fatty acid substrate specificity with different catalytic efficiencies and preferences in the presence of various fatty acid substrates. ${ }^{13}$ The first yeast n3Des gene cloned from Saccharomyces kluyveri showed a dramatic preference for LA over AA substrate, ${ }^{\mathbf{1 4 , 1 5}}$ and the same preference was found in the bifunctional $\Delta 12 / \mathrm{n} 3$ desaturases from Fusarium moniliforme, Fusarium graminearum, and Magnaporthe grisea. ${ }^{16}$ FADS15 and maw3 from Mortierella alpina also showed a strong preference for LA. ${ }^{17,18}$ In 2018, two new n3Des isolated 
from plants - ShFAD3 from Salvia hispanica and PfFAD3 from Perilla frutescens - were used for ALA production based on their C18 substrate preference. ${ }^{12}$ In contrast, the yeast n3Des gene Ppfad3 isolated from Pichia pastoris GS115 showed no substrate preference with nearly the same conversion rates towards each added substrate. ${ }^{19}$ Nevertheless, a new type of n3Des - sdd17 derived from an EPA-rich fungus Saprolegnia diclina and Pi-D17 from Phytophthora infestans - were found in their ability to exclusively desaturate C20 fatty acids. ${ }^{2021}$ Three new $\Delta 17$ desaturase enzymes isolated from Pythium aphanidermatum, Phytophthora sojae and Phytophthora ramorum were also reported with preferences for the C20 substrates. ${ }^{22,23}$

Although various $n-3$ fatty acid desaturases have been identified, few $\Delta 17$ Des have been isolated compared to reported $\Delta 15$ Des. In this study, we reported the two new $\Delta 17$ desaturases oRiFADS17 from Rhizophagus irregularis and oObFADS17 from Octopus bimaculoides. They were identified from the National Center for Biotechnology Information database using the known $\Delta 17$ desaturase oPaFADS17 gene (codon-optimized desaturase PaD17; GenBank accession numbers KT372000) as a template. Two additional hypothetical desaturase genes oAcFADS17 from Aplysia californica and oBgFADS17 from Biomphalaria glabrata - were chosen and synthesized altogether. To examine their activities and substrate preferences, all of them were expressed in Saccharomyces cerevisiae INVSc1. Based on bioinformatics analysis including topology model and molecular docking, the views of their structures, binding pockets and substrate tunnels were also clearly observed. The characterization and docking models of new $\Delta 17 \mathrm{Des}-$ oRiFADS17 and oObFADS17 - would contribute to elucidating the structure-function relationships of n3Des, and it would also provide a powerful new choice for genetic engineering of oleaginous organisms to produce the desired long-chain fatty acids in a sustainable way.

\section{Materials and methods}

\section{Strains and plasmids}

Escherichia coli Top 10 was supplied by our laboratory. The plasmid pYES2-oPaFADS17 was stored in Top $10 .{ }^{24}$ The oAcFADS17, oBgFADS17, oObFADS17 and oRiFADS17 genes were synthesized by Nanjing Genscript Biotechnology Co Ltd; $S$. cerevisiae yeast strain INVSc1 was obtained from Invitrogen (Shanghai, China); and plasmid pYES2/NT C (Invitrogen) was used for heterologous expression of these genes.

GenBank accession numbers. $R$. irregularis codon-optimized $\Delta 17$ desaturase oRiFADS17:MH028784.

O. bimaculoides codon-optimized $\Delta 17$ desaturase oObFADS17:MH028785.

\section{Media and cultivation conditions}

The isolated clones that contained these genes were cultivated in lysogenic broth medium with $100 \mu \mathrm{g} \mathrm{mL} \mathrm{m}^{-1}$ of ampicillin at $37{ }^{\circ} \mathrm{C}$ and shaken at $200 \mathrm{rpm}$. The yeast transformants were incubated with SC-U medium, which was synthetic minimal defined medium for yeast and contained $0.67 \%$ yeast nitrogen base, $2 \%$ raffinose or glucose, $0.01 \%$ amino acids (leucine, lysine, threonine, tryptophan, adenine, arginine and cysteine), $0.005 \%$ amino acids (aspartic acid, proline, serine, tyrosine, valine, histidine, isoleucine, methionine and phenylalanine) and $2 \%$ agar. Extra $2 \%$ galactose and $1 \%$ raffinose were supplemented during the induction period.

\section{Sequence comparison, phylogenetic analysis}

We used the amino acid sequence of oPaFADS17 as a template to BLAST these n3Des from the databases of the National Center for Biotechnology Information servers (https:// blast.ncbi.nlm.nih.gov/Blast.cgi). Four hypothetical desaturase proteins were then selected and compared with 16 other reported desaturases and aligned using the Clustal Omega web server, ver. 6. A GenBank accession number was provided for each sequence in Table 2 . They included a diverse superfamily of enzymes from various strains, including $M$. alpina 1S-4, $M$. alpina ATCC32222, F. moniliforme, $M$. grisea, $F$. graminearum, $S$. kluyveri, P. pastoris, C. elegans, P. frutescens, S. hispanica, S. diclina, $P$. aphanidermatum, $P$. ramorum, $P$. sojae, $P$. infestans, $P$. parasitica, $R$. irregularis, O. bimaculoides, B. glabrata and A. californica. The sequences underlined in red were three His-rich motifs.

Phylogenetic analysis showed the relationship among the four hypothetical proteins and 16 reported desaturases. The phylogenetic tree was developed using the substitution model, and gaps were treated using the method of pairwise deletion. This work was created with Microsoft Word, Clustal Omega, and Adobe Illustrator.

\section{PCR amplification and sequence analysis for oAcFADS17, oBgFADS17, oObFADS17 and oRiFADS17}

The oAcFADS17, oBgFADS17, oObFADS17 and oRiFADS17 genes were amplified by polymerase chain reaction (PCR) with forward primers and reverse primers including restrictive endonuclease sites EcoRI and XhoI, respectively (Table 1). The amplified PCR products were then subcloned into the vector pYES2/NT C, and the positive clones were selected and confirmed by DNA sequencing in both directions (Sunny Biotechnology Co Ltd, Shanghai, China).

Yeast transformation, heterologous expression in S. cerevisiae and determination of substrate preference

The constructed plasmids pYES2-oAcFADS17, pYES2oBgFADS17, pYES2-oObFADS17 and pYES2-oRiFADS17 were transformed into INVSC1 via the lithium acetate transformation method. On SC-U selective plates, the recombinant yeast clones that exhibited uracil prototrophy were selected and identified by colony PCR. The yeast transformant with a pYES2-oPaFADS17 vector was used as a control strain.

To acquire insight into the substrate specificities of these recombinant proteins, the transformants were cultured for $48 \mathrm{~h}$ at $20{ }^{\circ} \mathrm{C}$ in SC-U media. Various fatty acid substrates were supplemented into induction buffer with fatty acids (0.1 mM cis-LA, $0.1 \mathrm{mM}$ cis-GLA, $0.1 \mathrm{mM}$ cis-DGLA or $0.1 \mathrm{mM}$ cis-AA). Cells were collected via filtration by centrifugation for $30 \mathrm{~s}$ at 12000 
Table 1 Primers used in this study

\begin{tabular}{|c|c|c|c|}
\hline $\begin{array}{l}\text { Primer } \\
\text { name }\end{array}$ & $\begin{array}{l}\text { Restriction } \\
\text { enzyme }\end{array}$ & Oligo nucleotide sequence $\left(5^{\prime}-3^{\prime}\right)^{a}$ & Function \\
\hline Rid-F & EcoRI & CCGGAATTC ATGTCGCCCTTGGAGC & oRiFADS17 amplification for expression in S. cerevisiae \\
\hline Obd-F & EcoRI & CCGGAATTC ATGGTCACCGCTATCC & oObFADS17 amplification for expression in $S$. cerevisiae \\
\hline Obd-R & XhoI & CCGCTCGAG TTACTTGTACCAGTGGATGAGG & \\
\hline Oac-F & EcoRI & CCGGAATTC ATGGAGCCCACCCCCCCCGTCTCG & oAcFADS17 amplification for expression in $S$. cerevisiae \\
\hline Obg-R & XhoI & CCGCTCGAG TTACTTGTAGAGGTGG & \\
\hline
\end{tabular}

Table 2 Literature summary of catalytic efficiency of $n-3$ desaturases from various species with LA/GLA/DGLA and AA substrates

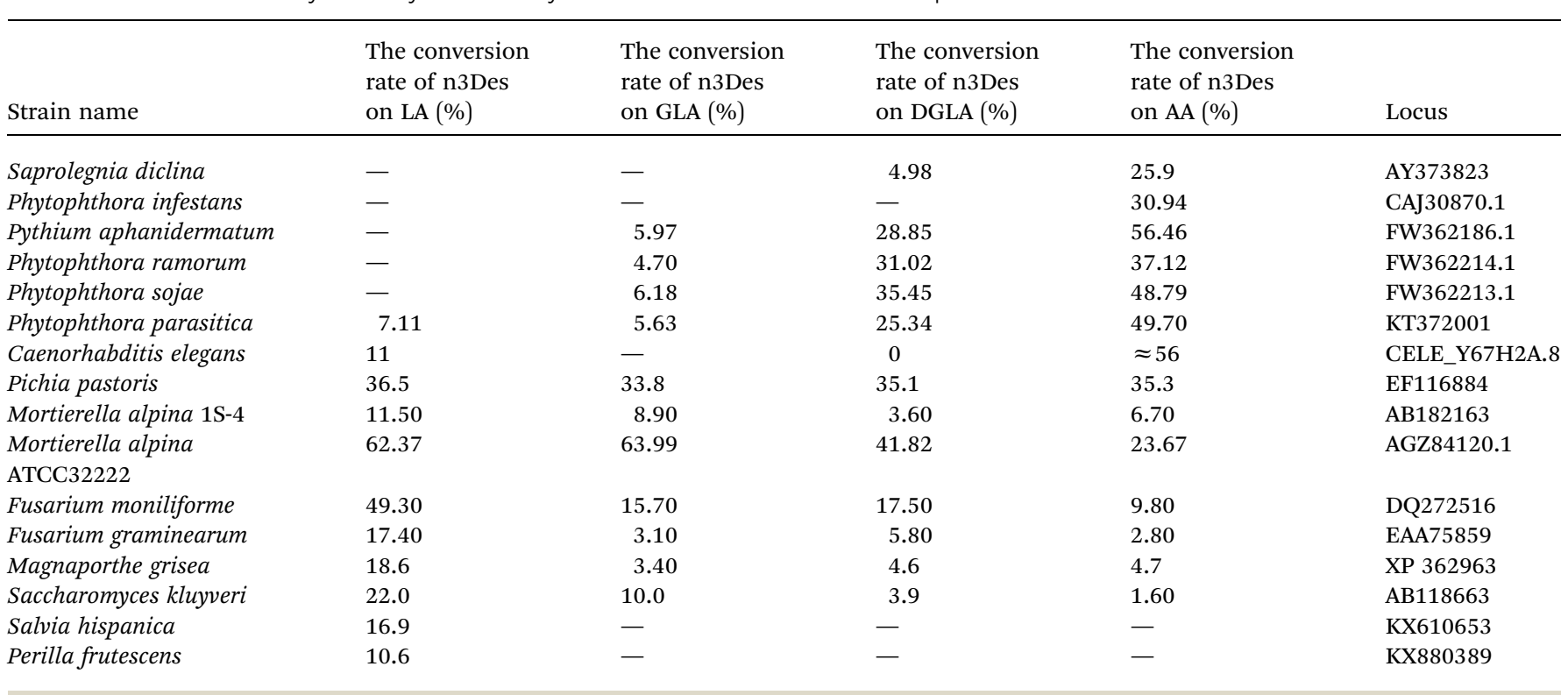

$\mathrm{g} \min ^{-1}$, washed twice with distilled water, and freeze-dried. The dried cells were used for fatty acid analysis and determination of substrate preference and the remainder was stored at $-80^{\circ} \mathrm{C}$.

\section{Western blotting}

To analyze the translation levels of these recombinant desaturases, $20 \mu \mathrm{L}$ of the collected cells were mixed with $5 \times$ SDS lysate buffer and heated in boiling water for $10 \mathrm{~min}$ for western blotting. The quantitative analysis method of bovine serum albumin was used to determine the volume of equivalent samples for SDS-PAGE gel loading. The gel was transferred to a polyvinylidene fluoride membrane (60 mA, $5 \mathrm{~h}$ ), which was then incubated with mouse THETM His tag antibody $(1: 5000)$ and special HRP-conjugated goat anti-mouse IgG $(1: 2000)$. Finally, the membrane was developed and fixed with the chemiluminescence reagent for $1 \mathrm{~min}$, and photographs were taken with FluorChem FC3.

\section{Lipid extraction and fatty acid analysis}

Lipid extraction and methyl esterification of free fatty acids was conducted as described previously. ${ }^{25}$ Fatty acid methyl esters
(FAMEs) profiles were then analyzed by GC-2010 (Shimadzu Co. Japan) with a $30 \mathrm{~m} \times 0.32 \mathrm{~mm}$ DB-wax column (Agilent) and an FID detector. The samples were measured with the following temperature program: $40{ }^{\circ} \mathrm{C}$ for $5 \mathrm{~min}$, increase to $120{ }^{\circ} \mathrm{C}$ at $20^{\circ} \mathrm{C} \mathrm{min}{ }^{-1}$, hold for $3 \mathrm{~min}$, ramp to $190{ }^{\circ} \mathrm{C}$ at $5{ }^{\circ} \mathrm{C} \mathrm{min}{ }^{-1}$, ramp to $220{ }^{\circ} \mathrm{C}$ at $4{ }^{\circ} \mathrm{C} \min ^{-1}$ and hold $220^{\circ} \mathrm{C}$ for $20 \mathrm{~min}$.

\section{Topology prediction}

Prediction of membrane-spanning helices and topology models for the four hypothetical proteins and oPaFADS17 were executed with the PolyPhobius program (https://omictools.com/ polyphobius-tool) HMMTOP (http://www.enzim.hu/hmmtop/ html/submit.html), TMHMM (http://www.cbs.dtu.dk/services/ TMHMM/) online websites and I-TASSER algorithms (https:// zhanglab.ccmb.med.umich.edu/I-TASSER/) (Fig. S1 and S2†). ${ }^{26}$

\section{Homology modeling of three-dimensional structures and molecular docking of oObFADS17 and oRiFADS17}

A template of crystal structure of human stearoyl-CoA hSCD1 desaturase was used to construct homology models of the oObFADS17 and oRiFADS17. The Schrödinger Suite shareware 
was used for molecular docking and the docking models were performed using PYMOL software (Fig. 7). Their models were docked with the representative substrates LA-CoA and AA-CoA. Two metal zinc-ions bound to the substrate-binding pockets were also depicted as grey or yellow spheres. The view of their predicted structures, binding pockets and substrate tunnels were clearly observed.

\section{Results}

\section{Biosynthetic process and literature summary of catalytic efficiency of n3Des}

Desaturases are large complex biological protein families that catalyze a wide range of fatty acids in the biosynthesis of PUFAs. Although they achieve these transformations via various biosynthetic processes $(\Delta 6 \mathrm{Des} / \Delta 6 \mathrm{Elo} / \mathrm{n} 3 \mathrm{Des}$ or $\Delta 9 \mathrm{Elo} / \Delta 8 \mathrm{Des} /$ n3Des), n3Des which converts $n-6$ polyunsaturates to their $n-3$ counterparts, is the key enzyme for the synthesis of long-chain fatty acids such as EPA and DHA (Fig. 1).,16

Some genes that encode n3Des were recently cloned and sequenced from various organisms. They consist mainly of $\Delta 15$ Des and $\Delta 17$ Des based on their substrate preferences and exhibit broad $n-6$ fatty acid substrate preferences and specificities. A review of the catalytic efficiencies of these reported n3Des is shown in Table 2, which illustrates their obvious preferences for C18 or C20 n-6 fatty acid substrates. FADS15 in M. alpina ( $\Delta 15$ desaturase $)$ had the highest catalytic activity towards LA, whereas oPaFADS17 ( $\Delta 17$ desaturase) had the highest catalytic activity towards AA.

\section{Sequence comparison, phylogenetic analysis}

The protein sequences of the four candidate $\Delta 17$ desaturase proteins from A. californica, B. glabrata, O. bimaculoides and $R$. irregularis had 416, 402, 419 and 397 amino acids, respectively. As illustrated in Fig. 2, sequence comparison analysis suggested that these ORFs shared greater sequence similarities and homologies to $\Delta 17$ desaturases than to $\Delta 15$ desaturases. The results also show that oRiFADS17 is $44 \%$ identical to Pi-D17, oObFADS17 is $46 \%$ identical to Ps-D17, oBgFADS17 is $45 \%$ identical to Pr-D17 and oAcFADS17 is $43 \%$ identical to oPaFADS17. The lighter shaded areas were conserved residues, and the blue shaded areas were consensus residues. These new genes were also predicted to have three typical His-rich desaturase motifs (underlined with red thick lines), suggesting they owned the characteristic of $\Delta \mathbf{1 7}$ desaturases.

Phylogenetic analysis also demonstrated the evolutionary relationship amongst these selected n3Des from various organisms using the neighbour-joining method (Fig. 3). According to the clear separation of the branches of these proteins identified by the phylogenetic tree, they were proposed to be roughly distinguished into different lineages: $\Delta \mathbf{1 5}$ desaturases, $\Delta 17$ desaturases and hypothetical $\Delta 17$ desaturases. As illustrated in Fig. 3, the last group comprised the four new genes. They were found from mycorrhizal fungi and sea mollusks, and were thus quite different from other n3Des. According to the bootstrap values at this branch point, the genome evolution of the phylogenetic relationships revealed significant differences in their species origins from the other reported $\Delta 17$ desaturases. In conclusion, it was deduced that they belonged to $\Delta 17$ desaturases.

\section{Cloning and expression of four candidate $\Delta 17$ desaturase genes in S. cerevisiae}

Owing to the bioinformatics analysis in the public databases, the oAcFADS17, oBgFADS17, oObFADS17 and oRiFADS17 genes were chosen and amplified by PCR with forward primers and

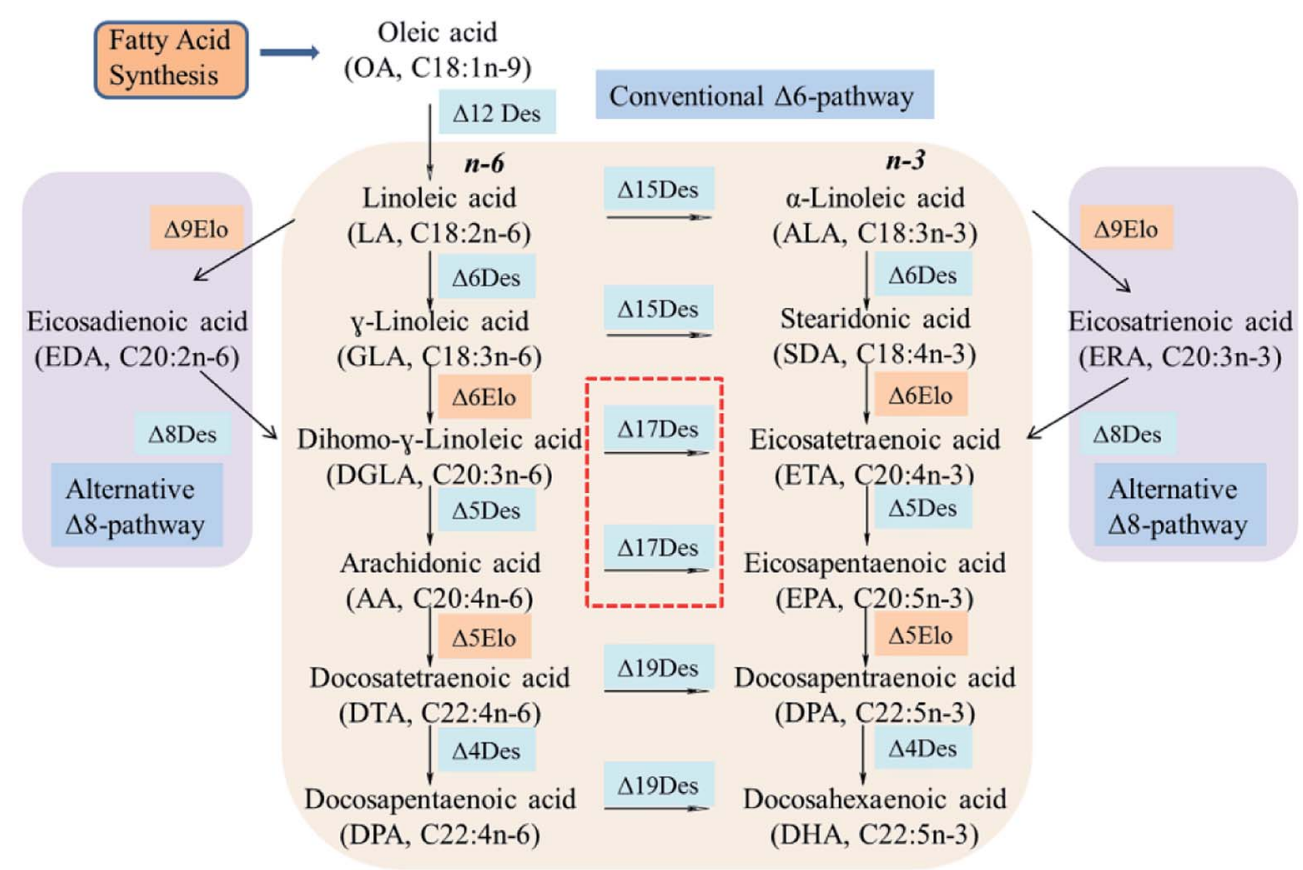

Fig. 1 Schematic diagram of the fatty acid pathways for EPA and DHA synthesis. 


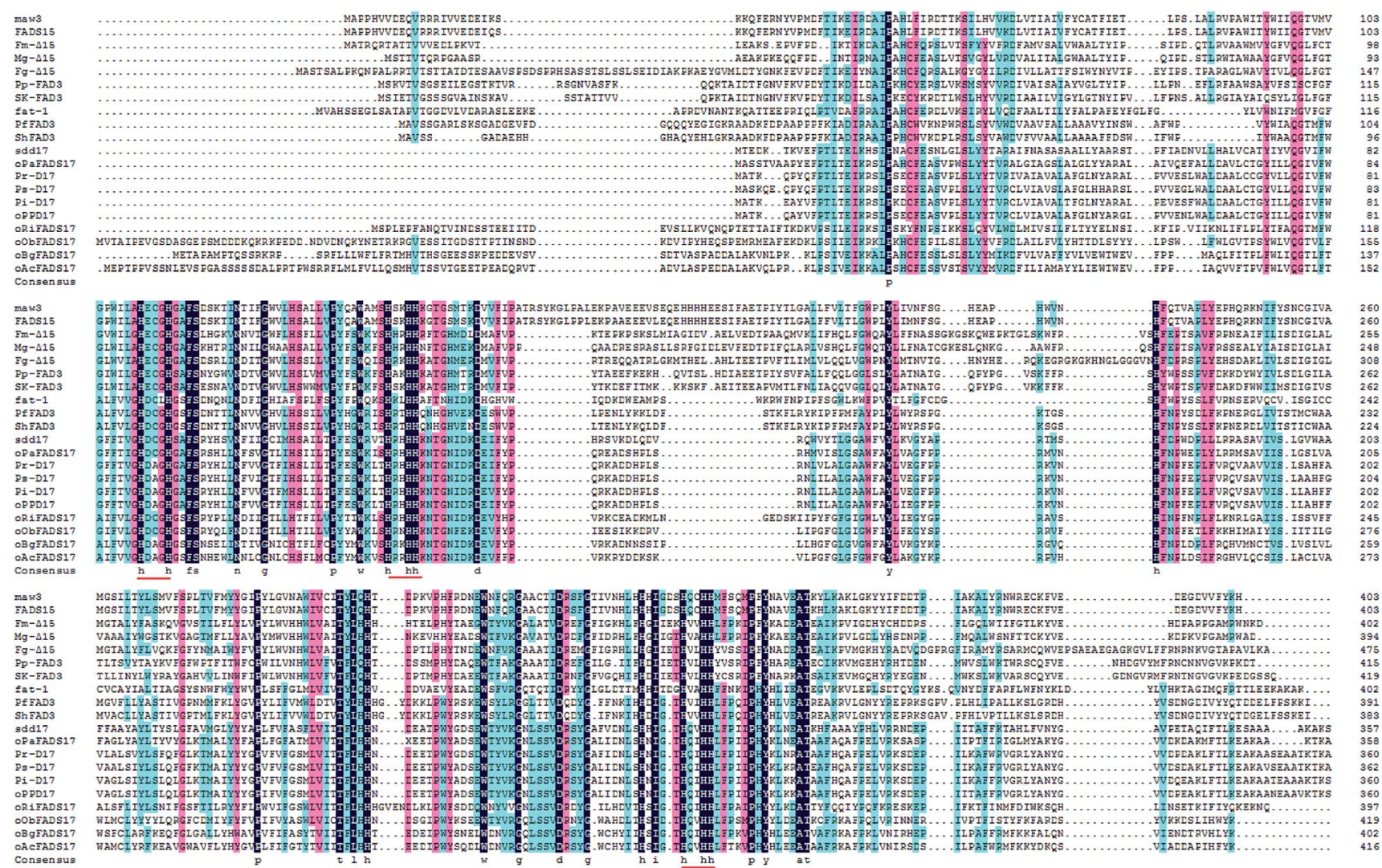

Fig. 2 Multiple sequence alignment of 16 reported $n-3$ fatty acid desaturases and 4 hypothetical desaturases.

reverse primers including the restrictive endonuclease sites EcoRI and XhoI, respectively (Table 1); these fragments were then subcloned into the vector pYES2/NT C (Fig. 4a). After lithium acetate transformation, their positive yeast clones heterologously expressed in INVSC1 were obtained. The result from the map of DNA agarose electrophoresis indicated that they were successfully expressed (Fig. 4b).
Western blot analysis of recombinant proteins was conducted to reveal successful transcription of the four candidate $\Delta 17$ desaturases and of the control, which was also a complementary proof for evaluation of transforming activity (Fig. 5a). The target $46 \mathrm{kD}$ bands on the membrane were marked with an arrow. The gray scanning analysis result showed no obvious differences among the translation levels of the recombinant proteins (Fig. 5b).

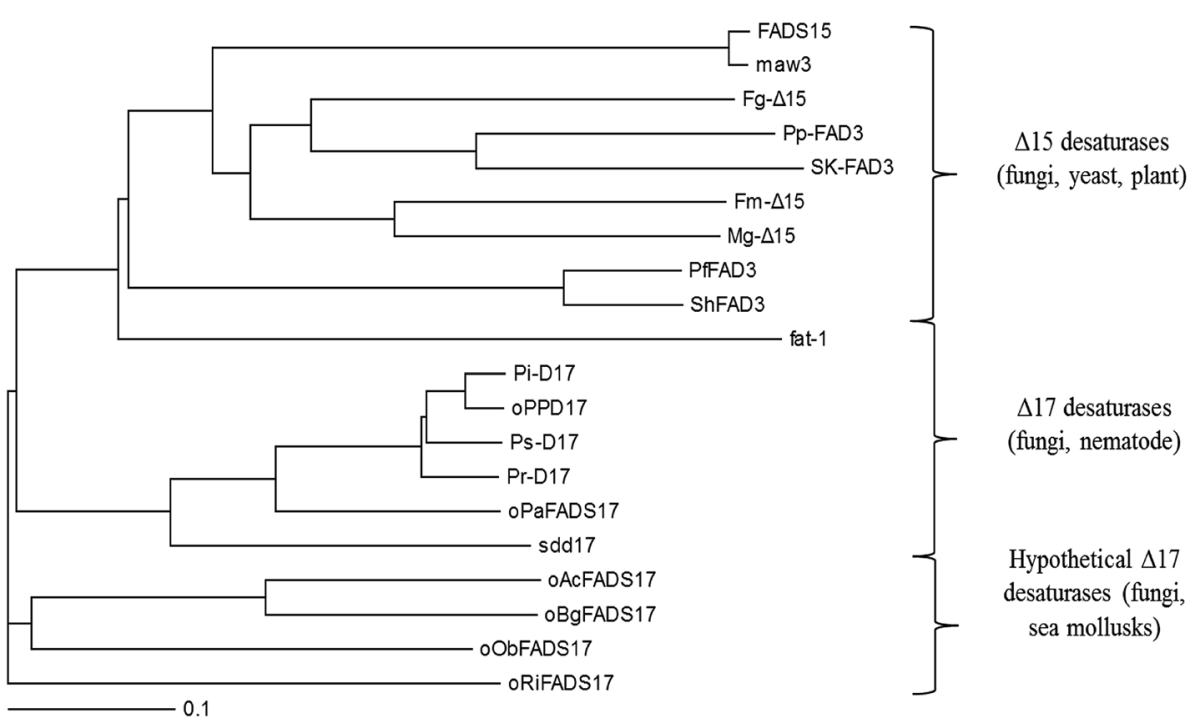

Fig. 3 Phylogenetic analysis showing the relationship among 16 selected desaturases and 4 hypothetical proteins. 
(a)

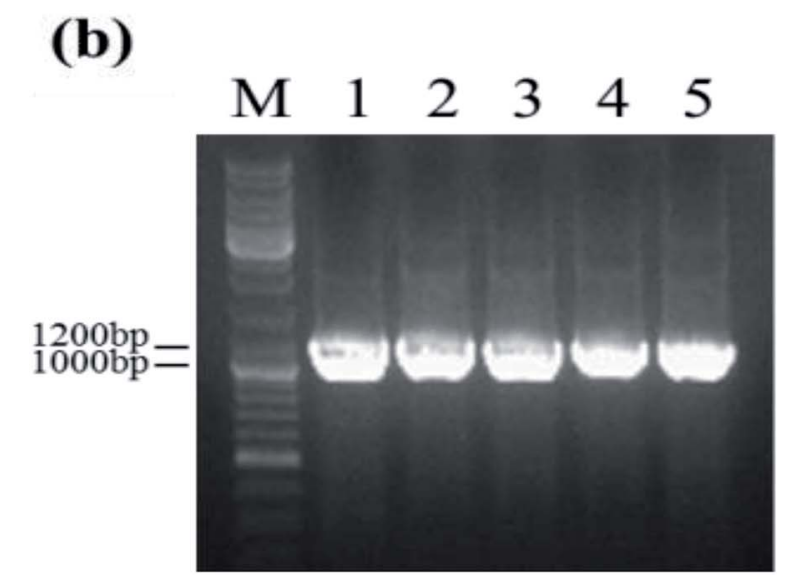

Fig. 4 Construction and verification of INVSC1 plasmids. (a) Construction of INVSC1 plasmids PYES2-OAcFADS17, pYES2oBgFADS17, pYES2-oObFADS17, pYES2-oRiFADS17. (b) Verification of INVSC1 transformants. M: maker; lane 1: control; lanes 2-5: pYES2OACFADS17, pYES2-oBgFADS17, pYES2-oObFADS17, pYES2oRiFADS17.

\section{Characterization of activities and substrate specificities of} four candidate $\Delta 17$ desaturases

The transformants were constructed to confirm that the four candidate proteins have $\Delta 17$ Des activity. Their lipids were extracted as previously described, ${ }^{25}$ and the fatty acids compositions of four candidate proteins were analyzed as shown in Table 3 and Fig. 6a. The fatty acid experiments indicated that the two $\Delta 17$ desaturases oRiFADS17 and oObFADS17 were capable of catalyzing LA, GLA, DGLA and AA conversion to ALA, SDA, ETA and EPA. Furthermore, they both exhibited stronger preference for C20 PUFA substrates DGLA and AA. The conversion rates of oRiFADS17 desaturase were $58.5 \% \pm 3.2 \%$ for AA and $35.1 \% \pm 1.6 \%$ for LA and those of oObFADS17 were $22.9 \%$ $\pm 2.6 \%$ and $3.8 \% \pm 0.2 \%$, respectively. According to the conversion rate in Fig. 1, oRiFADS17 desaturase in this study had higher conversion rate (3-4 fold) of the LA substrate than the control oPaFADS17 desaturase and other reported $\Delta 17$ desaturases, while containing a high conversion rate for AA (nearly 60\%) at the same time (Fig. 6b). In addition, oObFADS17 was found to be the first reported $\Delta 17$ desaturase from sea mollusks, although it converted little AA compared with oRiFADS17. However, another set of predicted $\Delta 17$ desaturase oAcFADS17 from A. californica and oBgFADS17 from $B$. glabrata did not have any detectable desaturase activity. Their fatty acid compositions of the oAcFADS17 and oBgFADS17 yeast transformants differed from those of the oPaFADS17 strain.

\section{Conversion rates in response to treatment at various temperatures}

The effects of temperatures on fatty acid substrate preferences and the transforming activities of some n3Des have been investigated. ${ }^{\mathbf{1 3 , 2 3 , 2 4 , 2 7}}$ To reveal the conversion rates in response to treatment at various temperatures, the yeast transformants were cultured $28{ }^{\circ} \mathrm{C}, 20{ }^{\circ} \mathrm{C}$ and $15{ }^{\circ} \mathrm{C}$. As shown in Table 4 , the conversion rates of the oPaFADS17, oObFADS17 and oRiFADS17 desaturase increased slightly as the incubation temperature decreased, but they did not change their substrate preferences after low-temperature treatment. Their responses were consistent with other n3Des such as maw3, FADS15 and CiFAD3, but not with oPPD17. ${ }^{13,23,24,27}$

\section{Topology prediction}

To further facilitate the gathering of biological information of these desaturases and explain the differences in the membrane structures of these proteins, topology analysis of the sequences was carried out with structural prediction software on Clustal Omega web servers. Their structural arrangements were very similar to that of the control protein oPaFADS17. As a result of the membrane topology in Fig. S1 and S2, $\uparrow$ their primary structures contained 2 large hydrophobic domains and 3 separate hydrophilic clusters but no confirmed important core cytb5 motif HPGG, unlike most $\Delta 6$ and $\Delta 9$ desaturases. Due to the high similarities in their amino acid sequences and membrane structures, they were predicted to show similar substrate chainlength and regioselectivity preferences. In contrast, extra AHs were predicted with a lower probability in oPaFADS17 (Cterminal) and oAcFADS17 (N-terminal). In addition, a signal peptide was found in the oBgFADS17 protein sequence $(\mathrm{N}$ terminal). Therefore, it was deduced that the structural diversities may be the reason why these two genes were inactive.

\section{Three-dimensional structures and molecular docking of oObFADS17 and oRiFADS17}

To further validate the molecular structures of substrates of oObFADS17 and oRiFADS17, their homology models of 3dimensional structures were also predicted. On the basis of a recent crystal structure of the human stearoyl-CoA desaturase hSCD1, the molecular docking of oObFADS17 and oRiFADS17 with LA-CoA or AA-CoA substrate was successfully performed (Fig. 7a, b, d and e). The carrier portion of the substrate and binding pocket were clearly observed. Two zinc-ions bound to the pocket center were close to the $n-6$ carbon atom of each 
(a)

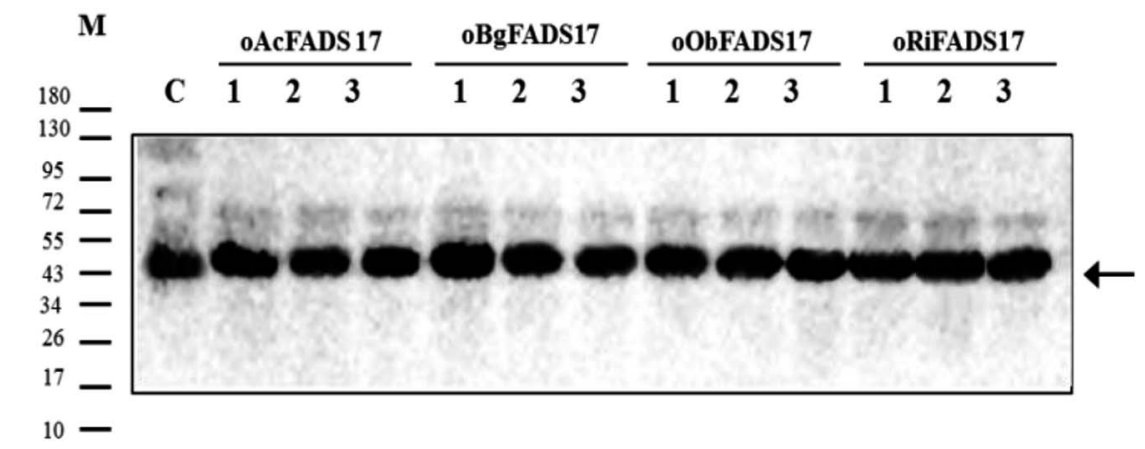

(b)

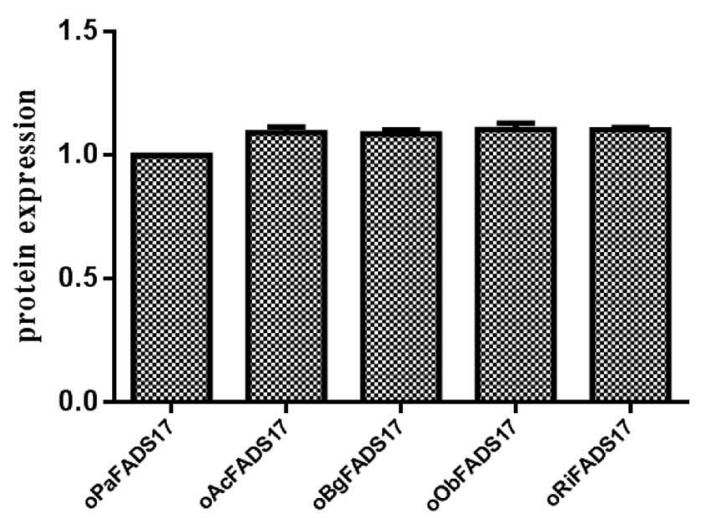

Fig. 5 Verification of translation levels of yeast transformants. (a) Western blotting of recombinant proteins expressed in S. cerevisiae. M: marker; C: control protein oPaFADS17; arrows in the western blotting indicated the location of the target protein. (b) Western-blot gray scale result showed that the average translation levels of 4 hypothetical $\Delta 17$ desaturases were almost the same amount.

substrate. From the kink and the narrow aperture of the substrate tunnel, it was assumed to present that how the substrate entered in or released out of the tunnel (Fig. $7 \mathrm{c}$ and f). Based on knowledge of these structures, the molecular mechanism of substrate specificity and preference would be further deeply investigated and this also enabled us to strengthen our understanding of the structure-function relationships of novel fatty acid desaturases.

\section{Discussion}

Several previous studies have reported substrate preferences and specificities in membrane-bound fatty acid desaturases such as $\Delta 4$ des $\Delta 6$ des, $\Delta 8$ des, $\Delta 9$ des and $\Delta 12$ des. $^{28-33}$ The biological information including sequence alignment, phylogenetic analysis and topology prediction was applied to help define the structure-function relationship of these desaturases.

Table 3 Fatty acid compositions (\% w/w) of the total lipid contents of yeast transformants expressing the control and pYES2-oAcFADS17, pYES2oBgFADS17, pYES2-oObFADS17, pYES2-oRiFADS17

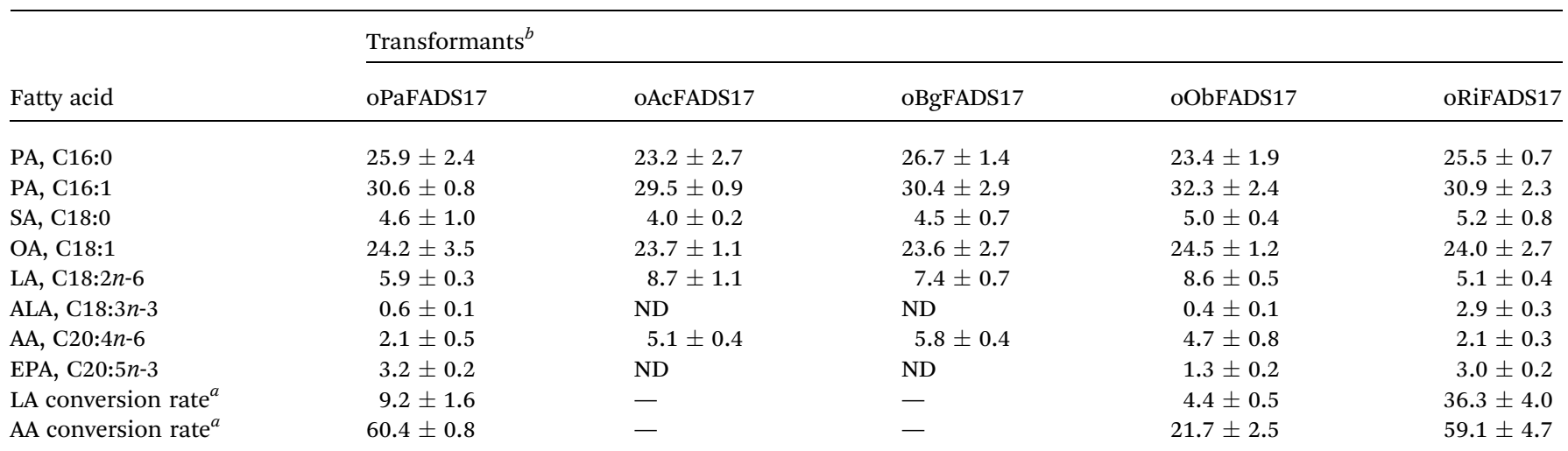

${ }^{a}$ Conversion rate $=100 \times[($ product $) /($ product + substrate $)]$. ND: not detected. ${ }^{b}$ Substrate concentration was 0.1 mM LA or AA. 
(a)

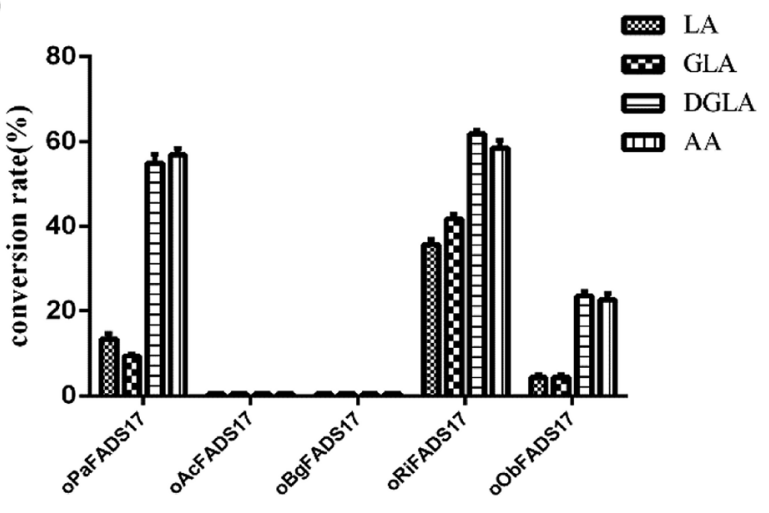

(b)

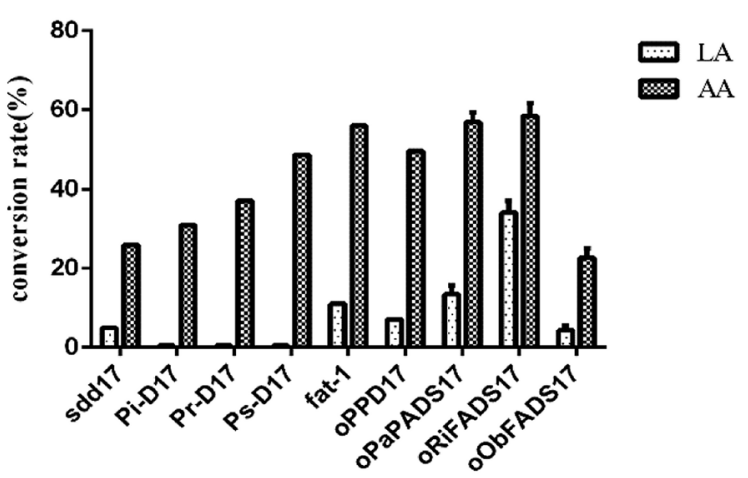

Fig. 6 Conversion rates of yeast transformants on different fatty acids. (a) Rate of substrate conversion of oPaFADS17, OAcFADS17, oBgFADS17, oObFADS17 and oRiFADS17 in S. cerevisiae. (b) Rate of substrate conversion of sdd17, Pi-D17, Pr-D17, Ps-D17, fat-1, oPaFADS17, oRiFADS17 and oObFADS17.

Based on the application of multiple sequence alignment, four candidate ORFs for $\Delta \mathbf{1 7}$ desaturase proteins from $A$. californica, B. glabrata, O. bimaculoides and $R$. irregularis were found. They were then compared and aligned with 16 reported n3Des with various substrate preferences. Pair-wise comparison showed that they all contained iron-binding sites with an active center and three typical His-rich motifs (one $\mathrm{HX}_{3} \mathrm{H}$ and two $\mathrm{HX}_{2} \mathrm{HH}$ ). Fig. 2 showed many lighter or darker shaded amino acid residues in their protein sequences, and a switch of even a single amino acid of these residues would result in a dramatic change in substrate preference and/or substrate characteristics. $^{29,30,33}$ These conserved residues between the $\Delta 15$ and $\Delta 17$ desaturases would be alternative possibilities for further examination of their activities and substrate preferences. ${ }^{24,29,31}$ Collectively, the residues provide significant insight into the relationship between the structure and function of n3Des.

Until now, the three-dimensional and crystal structure of n3Des have not been determined. A membrane topology model was used for determination of the functional domains using comparative analysis. ${ }^{34}$ On the basis of the high similarities in their amino acid sequences and the proposed topology models (Fig. S1 and S2 $\dagger$ ), proteins encoded by these genes were predicted to have $\Delta 17$ desaturase activity. However, this method is not entirely correct, such as with another two candidate $\Delta 17$ desaturase genes oAcFADS17 and oBgFADS17. It was assumed that the extra N-terminal AHs in oAcFADS17 and the N-terminal signal peptide assembly in the oBgFADS17 might cause them to lose their catalytic activities. Considering the high sequence and species similarities with oObFADS17, they deserve further investigation.

The typical types of n3Des have been reported from a wide range of species including microalgae, cyanobacteria, yeast, fungi and plants. ${ }^{6,22,34}$ The phylogenetic tree in Fig. 3 shows that the top group of n3Des was derived from $M$. alpina, yeast and plants. The second cluster consisted of $\Delta \mathbf{1 7}$ desaturases from Pythium and Phytophthora and from nematodes. Interestingly, the $\Delta \mathbf{1 7}$ desaturases gene fat- 1 from Caenorhabditis elegans was actually closer to the first category. ${ }^{35}$ This may be related to the source of species and genetic differences. The $\Delta 17$ desaturase oObFADS17 of the bottom group originated from sea mollusks together with oAcFADS17 and oBgFADS17, thus differed greatly from other n3Des. Although oAcFADS17 and oBgFADS17 could not catalyze each added $n-6$ fatty acid substrates, we believe that sea mollusks organisms are a new source of $\Delta 17$ Des. Characterization of oObFADS17 would not only deepen our knowledge of a natural source of n3Des, but also pave the way for elucidating of the molecular basis and the enigmatic enzyme mechanism.

According to the conversion rates (Table 3), the calculated conversion efficiencies of the three $\Delta 17$ desaturase enzymes PrD17, PsD17 and PaD17 were almost 6\% for the LA substrate. ${ }^{6}$ In this study, the oRiFADS17 desaturase had nearly $34 \%$

Table 4 Fatty acid compositions (\% w/w) of yeast transformants expressing pYES2-oPaFADS17, pYES2-oObFADS17, pYES2-oRiFADS17 plasmids at $15^{\circ} \mathrm{C}, 20^{\circ} \mathrm{C}$ or $28^{\circ} \mathrm{C}$

Conversion rate $^{a}(\%)$

$\underline{\mathrm{LA}, \mathrm{C} 18: 2 n-6 \quad \mathrm{AA}, \mathrm{C} 20: 4 n-6}$

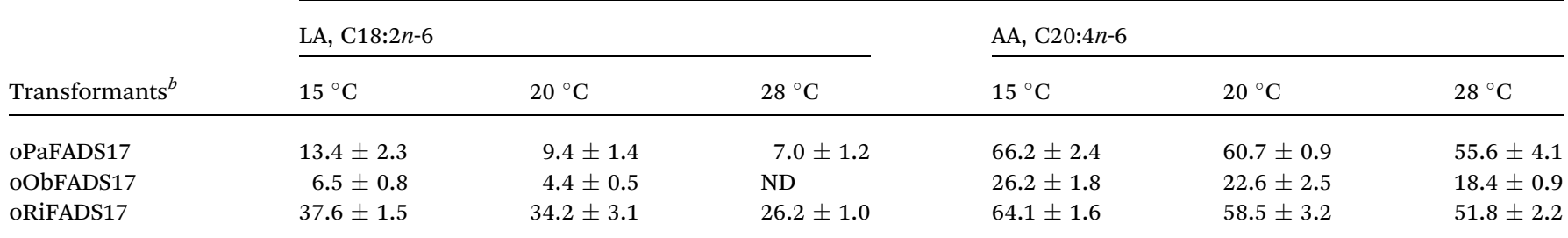

${ }^{a}$ Conversion rate $=100 \times[($ product $) /($ product + substrate $)]$. ND: not detected. ${ }^{b}$ Substrate concentration was 0.1 mM LA or AA. 
(a)

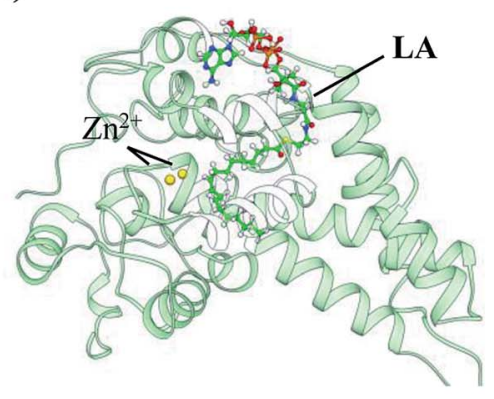

(d)

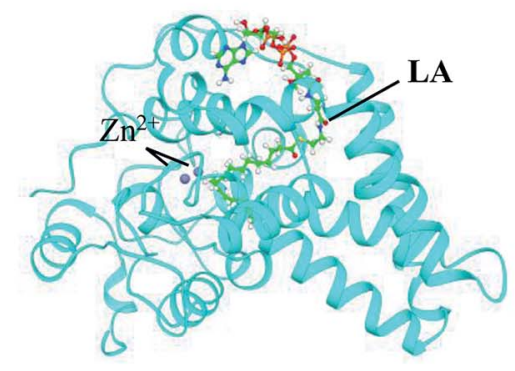

(b)

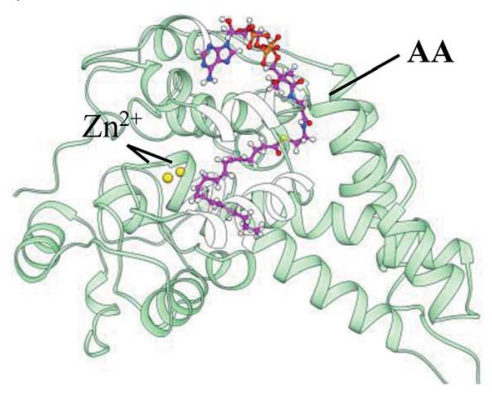

(c)

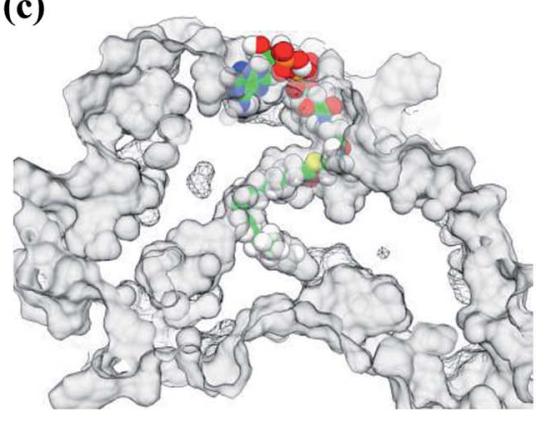

Binding pocket in oObFADS17 (e)

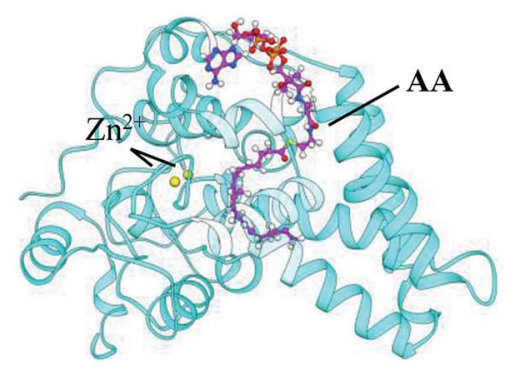

(f)

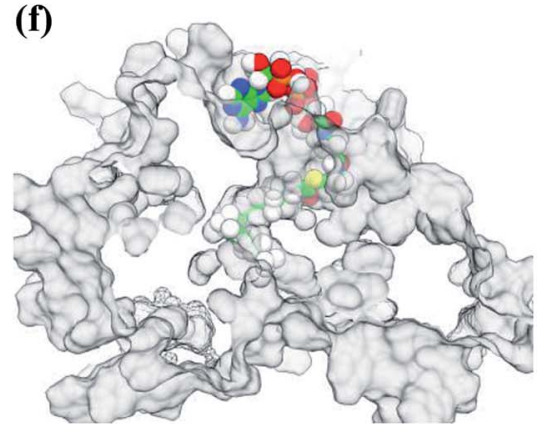

Binding pocket in oRiFADS17

Fig. 7 Homology modeling of three-dimensional structures and molecular docking of oObFADS17 and oRiFADS17.

conversion rate of the LA substrate (nearly 5 fold) and a higher conversion rate for AA (nearly $60 \%$ ) at the same time (Fig. 6b). The highest conversion rate for AA can reach $66 \%$ when $0.1 \mathrm{mM}$ AA was added at a lower temperature $15{ }^{\circ} \mathrm{C}$. Generally speaking, although the substrate preference of oRiFADS17 was weaker compared with other $\Delta 17$ desaturases, its comprehensive catalytic ability for $n$-6 PUFAs substrates increased significantly. Because of the strong preference for C18 substrates of endogenous n3Des or their limited catalytic efficiency in vivo in some oleaginous microorganisms, the newly identified $\Delta \mathbf{1 7}$ desaturase could be an excellent choice for genetic engineering of organisms and have great potential to be used to produce EPA and DHA. ${ }^{18,22,23}$

In contrast to the reported soluble acyl-ACP desaturases, little is known about the structure-guiding principle involved in substrate specificity and regioselectivity of n3Des, due to lack of the three-dimensional structure information. For the first time, the representative substrates LA-CoA and AA-CoA were chosen as the ligands, and the proposed structures of n3Des were clearly visualized based on molecular docking. Captures of the tunnel-like pockets were also shown in the hydrophobic interior of the n3Des. It was interpreted that how the substrate entered into or released from the tunnel, and this will help understand the crucial roles of active amino acids in regulating substrate specificity and enzyme activity.

Traditional source of $n-3$ PUFAs is diminishing due to overfishing and marine pollution, and a low dietary intake of $n-3$ PUFAs, especially EPA and DHA, is observed worldwide. Oleaginous microorganisms are increasingly attractive for their promising potential as an alternative source. In their fatty acid pathways for EPA and DHA synthesis, $\Delta 17$ Des is more important than $\Delta 15$ Des because its ability to directly desaturate C20 fatty acids substrates. The newly identified $\Delta 17$ desaturases could therefore contribute to producing PUFAs for dietary supplementation or the prevention and treatment of numerous diseases.

\section{Conclusion}

In this study, two genes - oRiFADS17 from $R$. irregularis and oObFADS17 from $O$. bimaculoides - were confirmed as new $\Delta 17$ desaturases and share high protein sequence similarities with known $\Delta 17$ desaturases. They show a preference for C20 PUFA substrates DGLA and AA, and the conversion rate of oRiFADS17 from AA to EPA was up to $60 \%$ when expressed in S. cerevisiae. Furthermore, oRiFADS17 desaturase has greater catalytic activity for LA substrates than other reported $\Delta 17$ desaturases. oObFADS17 is also the first reported $\Delta 17$ desaturase in a sea mollusk. The predicted 3-dimensional structures and molecular docking of oObFADS17 and oRiFADS17 with LA-CoA or AA-CoA substrate further supported our findings. The characterization of these two desaturases and the knowledge of their structures will contribute to strengthening our understanding of the relationship between the structure and function of novel fatty acid desaturases, and be of great value in PUFAs production for dietary supplementation and the prevention and treatment of numerous diseases. 


\section{Author's contributions}

CR designed the study, conducted the experiments and drafted the manuscript. $\mathrm{HC}$ and $\mathrm{YQC}$ helped propose and design the study, and revised the manuscript. HC, XT, ZG, JZ, HZ and WC assisted with revising the manuscript. All authors read and approved the final manuscript.

\section{Conflicts of interest}

The authors have no conflict of interest.

\section{Acknowledgements}

This study was supported by the National Natural Science Foundation of China (No. 31722041 and No. 31530056), the Fundamental Research Funds for the Central Universities (JUSRP51702A), the national first-class discipline program of Food Science and Technology (JUFSTR20180102), and the Jiangsu Province "Collaborative Innovation Center for Food Safety and Quality Control".

\section{References}

1 F. Echeverría, R. Valenzuela, M. C. Hernandez-Rodas and A. Valenzuela, Prostaglandins, Leukotrienes Essent. Fatty Acids, 2017, 124, 1-10.

2 S. Xia, J. Wang and J. Kang, Carcinogenesis, 2005, 26, 779.

3 J. Hirahashi, K. Kawahata, M. Arita, R. Iwamoto, K. Hishikawa, M. Honda, Y. Hamasaki, M. Tanaka, K. Okubo and M. Kurosawa, Sci. Rep., 2014, 4, 6406.

4 H. K. Maehre, J. Ida-Johanne, E. O. Elvevoll and E. Karl-Erik, Int. J. Mol. Sci., 2015, 16, 22636-22661.

5 J. Miyata and M. Arita, Allergol. Int., 2015, 64, 27-34.

6 Z. Xue, H. He, D. Hollerbach, D. J. Macool, N. S. Yadav, H. Zhang, B. Szostek and Q. Zhu, Appl. Microbiol. Biotechnol., 2013, 97, 1973-1985.

7 G. Vadivelan and G. Venkateswaran, BioMed Res. Int., 2014, 2014, 657414.

8 S. L. Pereira, A. E. Leonard and M. Pradip, Prostaglandins, Leukotrienes Essent. Fatty Acids, 2003, 68, 97-106.

9 C. M. Venegas, O. Sayanova and J. A. Napier, Prog. Lipid Res., 2010, 49, 108-119.

10 S. Eiji, A. Takahiro, I. Keita and S. Sakayu, Appl. Microbiol. Biotechnol., 2005, 66, 648-654.

11 A. Takahiro, S. Eiji, A. Takahiro, K. Hiroyuki, I. Yuji and S. Sakayu, J. Biosci. Bioeng., 2005, 69, 1021.

12 Y. Xue, B. Chen, A. N. Win, C. Fu, J. Lian, X. Liu, R. Wang, X. Zhang and Y. Chai, PLoS One, 2018, 13, e0191432.
13 H. Kikukawa, E. Sakuradani, S. Kishino, S. B. Park, A. Ando, J. Shima, M. Ochiai, S. Shimizu and J. Ogawa, J. Biosci. Bioeng., 2013, 116, 672-676.

14 T. Oura and S. Kajiwara, Microbiology, 2004, 150, 1983-1990. 15 T. Oura and S. Kajiwara, Biosci., Biotechnol., Biochem., 2008, 72, 3174-3179.

16 H. G. Damude, H. Zhang, L. Farrall, K. G. Ripp, J. F. Tomb, D. Hollerbach and N. S. Yadav, Proc. Natl. Acad. Sci. U. S. A., 2006, 103, 9446-9451.

17 E. Sakuradani, A. Ando, S. Shimizu and J. Ogawa, J. Biosci. Bioeng., 2013, 116(4), 417-422.

18 M. Wang, H. Chen, A. Ailati, W. Chen, F. H. Chilton, W. T. Lowther and Y. Q. Chen, Appl. Microbiol. Biotechnol., 2017, 102, 1-13.

19 X. Zhang, M. Li, D. Wei and L. Xing, Yeast, 2010, 25, 21-27. 20 Y. Chen, M. Zhang and G. Kemian, Biochem. Biophys. Res. Commun., 2010, 394, 158-162.

21 Y. Fu, X. Fan, X. Li, H. Wang and H. Chen, Appl. Biochem. Biotechnol., 2013, 171, 975-988.

22 H. Zhou and C. Wang, Exp. Ther. Med., 2017, 14, 2117-2125.

23 X. Tang, H. Chen, T. Mei, C. Ge, Z. Gu, H. Zhang, Y. Chen and W. Chen, Front. Microbiol., 2018, 14(9), 1878.

24 C. Rong, H. Chen, M. Wang, Z. Gu, J. Zhao, H. Zhang, W. Chen and Y. Chen, Appl. Microbiol. Biotechnol., 2018, 102(22), 9679-9689.

25 L. Wang, W. Chen, Y. Feng, Y. Ren, Z. Gu, H. Chen, H. Wang, M. J. Thomas, B. Zhang and I. M. Berquin, PLoS One, 2011, 6, e28319.

26 A. Roy, A. Kucukural and Y. Zhang, Nat. Protoc., 2010, 5, 725738.

27 P. Zhang, S. Liu, B. Cong, G. Wu, C. Liu, X. Lin, J. Shen and X. Huang, Mar. Biotechnol., 2011, 13, 393-401.

28 S. J. Gagne, D. W. Reed, G. R. Gray and P. S. Covello, Biochemistry, 2009, 48, 12298.

29 H. Shi, H. Chen, Z. Gu, Y. Song, H. Zhang, W. Chen and Y. Q. Chen, J. Lipid Res., 2015, 56, 2309-2321.

30 S. F. Li, L. Y. Song, G. J. Zhang, W. B. Yin, Y. H. Chen, R. R. Wang and Z. M. Hu, Biochem. Biophys. Res. Commun., 2011, 416, 165-171.

31 Z. L. Lim, T. Senger and P. Vrinten, Lipids, 2014, 49, 357-367. 32 D. Meesapyodsuk and X. Qiu, ACS Chem. Biol., 2014, 9, 922. 33 X. Zhang, D. Wei, M. Li, Y. Qi and L. Xing, Mol. Biol. Rep., 2009, 36, 567.

34 M. Wang, H. Chen, Z. Gu, H. Zhang, W. Chen and Y. Q. Chen, Appl. Microbiol. Biotechnol., 2013, 97, 1025510262.

35 L. An, Y. W. Pang, H. M. Gao, L. Tao, K. Miao, Z. H. Wu and J. H. Tian, Biochem. Biophys. Res. Commun., 2012, 428, 405410. 